\title{
SALUD MENTAL Y CARACTERÍSTICAS DE PERSONALIDAD DE LOS ESTUDIANTES UNIVERSITARIOS EN CHILE
}

\section{Introducción}

La educación superior ha experimentado un masivo incremento en su matrícula, tanto en nuestro país como en el resto del mundo. Sucesivas declaraciones de organismos internacionales plantean la necesidad de adecuar los sistemas educacionales a sus usuarios y hacen necesario preguntarse acerca de las características de los alumnos universitarios. Paradojalmente, éstas han sido poco exploradas en forma sistemática, especialmente desde la perspectiva de los problemas y dificultades que enfrentan los jóvenes ante un sistema masificado.

En este artículo nos referiremos a algunos estudios chilenos sobre esas características, desde una perspectiva de salud mental, así como a evidencia nacional acerca de estas mismas características en estudiantes de algunas carreras de la salud. Finalmente, mostraremos datos relativos a factores contextuales que pueden aumentar la vulnerabilidad a los bajos rendimientos o a la deserción académica.

\section{Los estudiantes en el sistema de educación superior}

De acuerdo con datos del Ministerio de Educación (MINEDUC), la matrícula total en el sistema de educación superior subió desde 172.996 estudiantes, en 1983, a 555.492, en 2004, triplicándose en 21 años. La matrícula de las universidades creció de 108.849 a 392.294 alumnos entre los mismos años, o sea que, por cada estudiante universitario en 1983, hay 3,6 alumnos el 2004. 
La matrícula de institutos profesionales ascendió de 25.244 a 104.844, es decir, aumentó 4,1 veces; mientras que la de centros de formación técnica sólo lo hizo desde 39.702 a 62.354, o sea, subió 1,5 veces.

Esto implica que de 15.116.435 habitantes con que cuenta el país, según la estimación de la Comisión Bicentenario, un 3,6\% están en el tercer nivel educacional. Aún más: de la población chilena estimada entre 18 y 24 años, el porcentaje en universidades ha aumentado desde 16,32\% a 33,22\%, entre 1992 y 2002, según la misma fuente. La distribución por sexo del total del sistema fue cercana, con un leve predominio de hombres (303.399) sobre mujeres (280.560). La distribución por quintil de ingreso es poco equitativa, al concentrarse mayores porcentajes en los quintiles de altos ingresos (donde un 74\% llega al nivel universitario versus un $15 \%$ en el quintil opuesto).

\section{La salud mental del estudiante universitario}

Como diversos estudios han demostrado en la última década, la prevalencia de problemas emocionales es elevada entre los chilenos. El más completo de ellos ha sido el Estudio Chileno de Prevalencia Psiquiátrica (ECPP), realizado por un equipo en la Universidad de Concepción (Vicente, 2002). Es éste un estudio diseñado para representar a la población adulta del país, basado en una muestra aleatoria, estratificada en dos etapas, que consideró viviendas y, posteriormente, personas de 15 años o más. Santiago, Concepción, Iquique y Cautín fueron las provincias elegidas, cada una perteneciente a una región diferente del país y estimada representativa de la población nacional. El estudio, conducido por el Departamento de Psiquiatría y Salud Mental de la Universidad de Concepción, entre julio de 1992 y junio de 1999, encontró que la prevalencia de vida de trastornos mentales para la población chilena fue de $32 \%$, y de 2,4\% considerando los últimos seis meses. La población joven tuvo 
índices comparativamente bajos, presentando más patologías relacionadas con el consumo de alcohol y drogas. La tabla 1 muestra que, en la población comprendida entre los 15 y los 24 años, los trastornos afectivos, ansiosos y esquizofrénicos tuvieron tasas cercanas a las del total de la población estudiada, pero, también, que las personalidades antisociales y el consumo de alcohol y drogas fueron más elevados. En el estudio de Vicente, los niveles educacional y socioeconómico protegieron de patología psiquiátrica: es decir, a mayor nivel de estudios, menor riesgo relativo de presentar psicopatología.

Tabla 1. Proyección del número de estudiantes universitarios con problemas emocionales, derivada de estudio de prevalencia de trastornos mentales en Chile, población total y la comprendida entre los 15 y 24 años (Vicente, 2002).

\begin{tabular}{|l|c|c|c|}
\hline \multirow{2}{*}{ Diagnóstico } & Total & $15-24$ & Proyección \\
\cline { 2 - 4 } & Porcentaje & Porcentaje & $\begin{array}{c}\text { Total } \\
555.492\end{array}$ \\
\hline Trastorno Afectivo & 16,29 & 13,86 & 76.991 \\
\hline Trastorno Ansioso & 12,55 & 11,30 & 62.771 \\
\hline Alcohol - Drogas & 11,00 & 15,31 & 85.046 \\
\hline Esquizofrenia & 2,20 & 2,18 & 12.110 \\
\hline Trastorno Estrés Postraumático & 4,03 & 3,21 & 17.831 \\
\hline Personalidad Antisocial & 0,95 & 1,45 & 8.055 \\
\hline Trastorno Orgánico & 4,40 & & 8.111 \\
\hline
\end{tabular}

Si proyectamos los hallazgos del ECPP a la población universitaria chilena el 2004 (tabla 1), debiera haber un total de 76.991 universitarios con cuadros afectivos, 62.771 con cuadros ansiosos, 85.046 con problemas de alcohol y drogas, 12.110 con esquizofrenia, 17.831 con trastorno de estrés postraumático, 8.055 con personalidad antisocial y 8.111 con cuadros neuropsiquiátricos orgánicos. Esto representa un problema importante de salud pública.

Un estudio reciente corrobora los datos anteriores acerca de la salud mental de los estudiantes universitarios (Santander 
2004). Se analizó allí, sistemáticamente, la relación entre salud mental y rendimiento académico. Para ello se comparó estudiantes en riesgo de desertar del sistema académico, que solicitaron atención en el Servicio Médico Estudiantil de una universidad, con un grupo control de estudiantes que no habían tenido problemas para mantener la continuidad de sus estudios. A través de una regresión logística, se identificaron las variables que predecían un mayor riesgo. Se encontró que un 62,3\% de los casos presentaban un diagnóstico de depresión, en comparación con un 11,3\% de los controles $(\mathrm{p}<0,001)$. Adicionalmente, un $80,1 \%$ de los casos presentaba algún tipo de disfunción familiar, en comparación con un 33,9\% de los controles ( $p<0,0001)$.

Estos hallazgos se hicieron en un conjunto de universitarios que provenían de estratos socioeconómicos medios y bajos: un $71,6 \%$ de los casos y un 35,8\% de los controles se encontraban en los quintiles más bajos de ingresos. Al analizar en mayor detalle las variables estadísticamente significativas que diferenciaban a ambos grupos, se encontró que, si bien ambos grupos presentaban consumo de sustancias químicas (alcohol y sustancias ilegales), no había mayor diferencia entre casos y controles. Es digno de mención que el total del universo estudiado consumía más que la población general del mismo grupo etario. La comorbilidad más frecuente entre los casos fue una condición médica (la hipertensión arterial), lo que apunta a un índice de vulnerabilidad global para los casos: el menor rendimiento académico, la depresión y los problemas biomédicos tienden a concentrarse en los grupos de mayor vulnerabilidad psicosocial.

Otro hecho llamativo en el estudio de Santander fue que el riesgo aumentó entre hijos de mujeres profesionales: en el grupo casos un 34\% de las madres ejercían su profesión universitaria, mientras que en los controles lo hacía el 9\% ( $\mathrm{p}<0,002)$. Esto en una población, como ya dijimos, proveniente de estratos socioeconómicos en desventaja. 


\section{Inventarios de personalidad en estudiantes universitarios}

Existen múltiples estudios sobre personalidad de estudiantes universitarios, fundamentalmente porque este es el grupo utilizado más frecuentemente para la validación de los inventarios de personalidad. Uno de los estudios más realizados, desde el punto de vista de selección de personal, es el Myers-Briggs Type Indicator (Hirsch y Kummerow, 1998), que mide las preferencias en cuatro escalas derivadas de la teoría de tipos psicológicos de Carl Jung. En ésta, las personas son seleccionadas en sus preferencias de acuerdo con:

- Introversión (I), con sus intereses centrados en un mundo interior de conceptos e ideas, versus extroversión (E), con intereses centrados en el mundo externo de las acciones, objetos y personas.

- Sentir (S), tendientes a percibir hechos inmediatos, prácticos y de la vida real, versus intuir $(\mathrm{N})$, tendientes a percibir posibilidades, relaciones y significados de las experiencias.

- Pensar ( $\mathrm{T}$ ), tendientes a hacer juicios sobre decisiones objetiva e impersonalmente, versus emocionar (F), tendientes a hacer juicios subjetiva y personalmente.

- Juzgar (J), tendientes a vivir de un modo planeado y decidido, versus percibir $(\mathrm{P})$, tendientes a vivir de un modo espontáneo y flexible.

Un tipo individual se expresa en una de dieciséis combinaciones posibles de esas preferencias. Por ejemplo, un ENTP tendría una preferencia por la extraversión, intuición, pensamiento y percepción. Por otra parte, la preferencia por cada categoría puede ir desde leve a fuerte.

Se ha estudiado los efectos del tipo de personalidad, según Myers-Briggs, y los rendimientos estudiantiles (Felder y cols., 2002), encontrando que los tipos I, P y J tendían a tener mejores rendimientos y a graduarse antes, y que los logros podían modificarse utilizando una estrategia experimental enfatizando 
la interacción en pequeño grupo y un proceso de aprendizaje activo.

Tabla 2. Frecuencia de tipos psicológicos en estudiantes y profesionales chilenos. Muestra de población general, profesionales de salud ( $\mathrm{n}=124)$, alumnos de IV Enfermería (n=75) y II Medicina $(\mathrm{n}=117)$.

\begin{tabular}{|l|c|c|c|c|}
\cline { 2 - 5 } \multicolumn{1}{c|}{} & Pob. Gral. & Prof. de salud & IV Enfermería & II Medicina \\
\hline Extroversión (E) & $75 \%$ & $67 \%$ & $58 \%$ & $64 \%$ \\
\hline Introversión (I) & $25 \%$ & $33 \%$ & $39 \%$ & $36 \%$ \\
\hline Sensación (S) & $75 \%$ & $53 \%$ & $63 \%$ & $54 \%$ \\
\hline Intuición (N) & $25 \%$ & $47 \%$ & $36 \%$ & $46 \%$ \\
\hline Pensamiento (T) & $50 \%$ & $75 \%$ & $33 \%$ & $46 \%$ \\
\hline Sentimiento (F) & $50 \%$ & $25 \%$ & $65 \%$ & $54 \%$ \\
\hline Juicio (J) & $50 \%$ & $90 \%$ & $86 \%$ & $85 \%$ \\
\hline Percepción (P) & $50 \%$ & $10 \%$ & $14 \%$ & $15 \%$ \\
\hline
\end{tabular}

Nosotros hemos utilizado la versión de Kersey del inventario de Myers-Briggs, en una adaptación chilena (Florenzano, 2003), aplicándola a diversos grupos de estudiantes y profesionales de universidades de la Región Metropolitana. En sucesivos estudios encontramos, como se aprecia en la tabla 2, que los profesionales de la salud encuestados presentaban moderadamente más tipos I (33 versus $25 \%$ ) que la población general, pero, pronunciadamente, más tipos N (47 versus 25\%), T (75 versus 50\%) y, sobre todo, tipos $\mathrm{J}$ (90 versus $50 \%$ ). Al comparar a los estudiantes de medicina y enfermería con la población general y con grupos de profesionales egresados, se puede apreciar que, entre ellos, predominan el subtipo "idealista" (NF) con 25 ó 27\%, en comparación al 12\% en población general, y $15 \%$ entre los profesionales. Los "racionales" (NT) son más entre los estudiantes de medicina (21\%) que de enfermería (11\%), quienes están muy cerca de la población general (12\%). Entre los profesionales, los NT llegan a un 30\%. Entre los estudiantes de enfermería predominan los "guardianes" (SJ) con un $60 \%$, sobrepasando a los estudiantes de medicina (49\%), quienes están cerca de los profesionales de la salud (53\%). Todos estos últimos están por sobre el porcentaje de SJ de la población 
general (38\%). Lo mismo sucede en el cuarto tipo, los "artesanos" (SP), donde los estudiantes están cerca entre sí (5\% medicina y $4 \%$ enfermería) versus $1,3 \%$ de los profesionales y $38 \%$ de la población general.

\section{Conclusiones}

En el planeamiento de la Reforma Educacional, en general, y en el sector de educación superior, en particular, es importante considerar las características de los educandos. Éstos tienen, de acuerdo a la evidencia actual, problemas importantes de salud mental, que provienen, por una parte, de las altas tasas de prevalencia de trastornos emocionales de todos los chilenos (Araya y cols., 2003) y, por otra, de la situación especial que enfrentan en un sistema educacional complejo y de cambio rápido. Dentro de estos problemas, es especialmente preocupante la frecuencia de las consecuencias del consumo de alcohol y sustancias químicas, sobre todo cuando se da en profesionales que trabajarán con personas, sea cuidándolas o como formadores del futuro recurso humano.

Los datos de Santander apuntan al rol de la familia en el riesgo de depresión y en los problemas de salud mental entre estudiantes universitarios. La correlación entre funcionamiento familiar y rendimiento académico, encontrada por Santander, ha sido dada a conocer antes para todo el sistema escolar: un estudio (Camhi y Arbola, 2004) muestra cómo las expectativas y la composición familiares influyen en los resultados de Lenguaje y Matemáticas de la pruebas SIMCE. Como exhibe la tabla 3, las familias completas son un factor protector; del mismo modo, en los niveles socioeconómicos más en desventaja, también lo es la presencia de la madre en el hogar. 
Tabla 3. Puntaje promedio SIMCE 2003, para $2^{\circ}$ medio, según conformación del hogar. (Instituto Libertad y Desarrollo, 2005).

\begin{tabular}{|l|c|c|c|c|c|c|}
\cline { 2 - 7 } \multicolumn{1}{c|}{} & \multicolumn{2}{c|}{ Puntaje promedio } & \multicolumn{3}{c|}{ Diferencia de puntajes* } \\
\hline \multicolumn{1}{c|}{ Clasificación } & Lenguaje & Matemáticas & \multicolumn{2}{c|}{ Lenguaje } & \multicolumn{2}{c|}{ Matemáticas } \\
\hline Vive con la madre y el padre & 256,3 & 250,9 & 0,0 & NS & 0,0 & NS \\
\hline $\begin{array}{l}\text { Vive sólo con la madre, sin } \\
\text { abuelos ni otros }\end{array}$ & 249,7 & 240,1 & 6,6 & $\mathrm{~S}$ & 10,8 & $\mathrm{~S}$ \\
\hline $\begin{array}{l}\text { Vive con la madre, abuelos } \\
\text { y/u otros }\end{array}$ & 254,4 & 244,3 & 1,9 & $\mathrm{NS}$ & 6,6 & $\mathrm{~S}$ \\
\hline $\begin{array}{l}\text { Vive sólo con el padre sin } \\
\text { abuelos ni otros }\end{array}$ & 244,1 & 234,8 & 12,2 & $\mathrm{~S}$ & 16,1 & $\mathrm{~S}$ \\
\hline $\begin{array}{l}\text { Vive con el padre, abuelos } \\
\text { y/u otros }\end{array}$ & 250,7 & 241,8 & 5,6 & $\mathrm{~S}$ & 9,1 & $\mathrm{~S}$ \\
\hline $\begin{array}{l}\text { Vive con abuelos u otros, } \\
\text { sin el padre ni la madre }\end{array}$ & 239,5 & 227,6 & 16,8 & $\mathrm{~S}$ & 23,3 & $\mathrm{~S}$ \\
\hline
\end{tabular}

* La diferencia de puntajes y su validez estadística se ha comparado respecto de quienes viven con ambos padres.

La personalidad de los alumnos debe estudiarse más sistemáticamente, lo que no es difícil dado el número de inventarios de personalidad que han sido validados en Chile utilizando poblaciones estudiantiles como sustrato. En el caso que hemos analizado, utilizando la clásica tipología junguiana operativizada por Myers-Briggs, encontramos que los profesionales tienden a hacer más evaluaciones utilizando diversos canales preceptuales (N) que la población general, donde predomina el uso de aproximaciones únicas (S). Asimismo, los profesionales muestran mayor racionalidad (T) que la población general. Los estudiantes están en un nivel intermedio al respecto, con un predominio del sentimiento entre los alumnos de enfermería y del pensamiento en los de medicina. Asimismo, la función J es mucho más frecuente entre los profesionales de la salud (90\%) que en la población general (50\%), función que es también predominante en los estudiantes de dichas carreras. Este tipo de análisis puede deberse a la selección de los estudiantes más racionales y más capaces de hacer juicios 
prudenciales que realiza el sistema de acceso a las universidades (PSU): la correlación entre rendimiento académico en la enseñanza media, la admisión a las universidades y los rendimientos dentro de las universidades es elevada en estudios internacionales, que muestran que lo primero predice lo tercero (Folder, 1993).

La influencia de la personalidad y de los problemas de salud mental pasa a ser pues una consideración relevante en un sistema que, a diferencia de lo que aconteció históricamente, ya no incorpora solamente a los segmentos de mejor rendimiento del sistema secundario, sino que se ha masificado, dando formación universitaria, técnica o profesional a cantidades importantes de jóvenes. Esta situación, que se dio en el mundo anglosajón en la mitad del siglo pasado, generó estudios en profundidad y la organización de un sistema de salud mental en los campi de las universidades (Glasscote y Fishman, 1973).

\section{Gráfico 1}

Comparación de tipos psicológicos entre población general, estudiantes y profesionales de carreras de Salud (Santiago, 2003), $\mathrm{n}=432$.

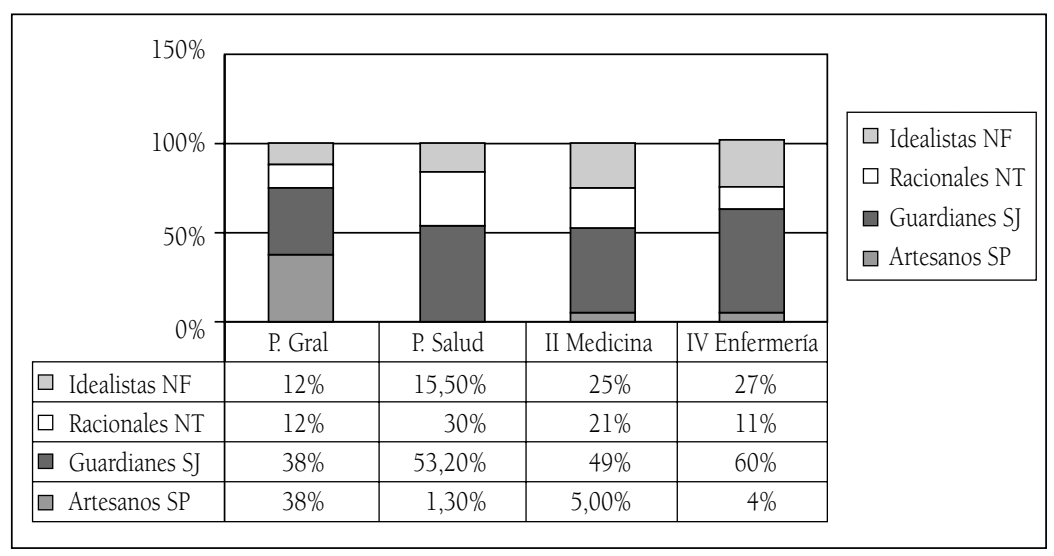




\section{Referencias bibliográficas}

Araya, R., Lewis, G.H., Rojas, G., Mann, A.H. "Patient Knows Best"detection of common mental disorders in Santiago, Chile: cross sectional study. British Medical Journal, v. 322, pp. 79-81.

Camhi, R. y Arbola, M.E. Familia y logros escolares. Libertad y Desarrollo, Informe Social n. 92, junio de 2005.

Felder, R.M., Felder, G.N. y Dietz, E.J. The Effects of Personality Types on Engineering Student Performance and Attitudes. Journal of Engineering Education, v. 91, n. 1, 2002, pp. 3-17.

Folder, R.M., Forrest, L., Baker-Ward, E.J. \& Mohr, P.H. A Longitudinal Study of Engineering Students Performance and Retention. Journal of Engineering Education, 1993, v. 82, n. 1, pp. 15-21.

Glasscote, R. y Fishman, M.E. Mental Health on the Campus: A field study. Joint Information Service of the American Psychiatric Association and National Association for Mental Health, Washington DC, 1973.

Hirsch, S. y Kummerow, J. Tipos de Personalidad. Paidós, Barcelona, 1998.

Kersey, R. Please Understand Me II: Temperament, Character, Inteligence. Prometheus, Nemesis Books, Del Mar, CA, 1998.

Ministerio de Educación, Chile. Matrícula de Pregrado para los años 1983 a 2004. Disponible en http://w3app.mineduc.cl/edusup/pags/comp_ matr_educ_sup.html

Santander, S. Cómo afectan los problemas de salud y factores y conductas de riesgo el rendimiento académico en alumnos universitarios. Tesis de Magíster en Adolescencia, Universidad del Desarrollo, 2004.

Vicente P., B., Rioseco, P., Saldivia, S., Kohn, R. y Torres, S. Estudio chileno de prevalencia de patología psiquiátrica (DSM-III-R/CIDI) (ECPP). Revista Médica de Chile, v. 130, n. 5, Santiago, mayo 2002. 